\title{
WILEY-VCH
}

DOI: $10.1002 /(($ please add manuscript number $))$

Article type: Communication

\section{Microscopic mechanism of doping-induced kinetically-constrained crystallization in phase-change materials}

Tae Hoon Lee*, Desmond Loke, and Stephen R. Elliott*

\author{
Dr. T. H. Lee, Prof. S. R. Elliott \\ Department of Chemistry \\ University of Cambridge \\ Lensfield Road, Cambridge, CB2 1EW, UK \\ E-mail: th132@cam.ac.uk, $\underline{\text { sre1@cam.ac.uk }}$ \\ Dr. D. Loke \\ Department of Engineering Product Development \\ Singapore University of Technology and Design \\ 8 Somapah Road, Singapore 487372, Singapore
}

Keywords: Phase-change materials, doping, kinetically-constrained crystallization, structural heterogeneity, DFT calculation

Doping of materials is enormously diverse in its purpose, covering the amelioration of electrical, ${ }^{[1]}$ magnetic, ${ }^{[2]}$ or optical ${ }^{[3]}$ properties of materials. Doping is of equal significance for glass science, since it is a simple, yet extremely effective, method to modulate the crystallization kinetics of, in particular, "bad" glass formers, such as (semi-) metals, ${ }^{[4,5]}$ metallic glasses, ${ }^{[6]}$ or phase-change (PC) materials. ${ }^{[7]}$ PC materials show ultrafast speeds of crystallization on the order of $\sim 1 \mathrm{~ns}$, or even sub-nanoseconds,${ }^{[8]}$ together with other unique characteristics, which renders them very promising for the development of devices for, e.g., so-called universal-memory, logic,,$^{[9,10]}$ or neuromorphic-computing ${ }^{[11,12]}$ applications, to replace current silicon-based technologies. However, the tendency of (unwanted) spontaneous crystallization of the corresponding amorphous phases at elevated temperatures diminishes long-term (amorphous-phase) data retention, imposing a substantial limitation in diverse practical applications. Dopants, notably $\mathrm{N},{ }^{[13-18]}$ have been shown significantly to improve not only such a thermal-retention property of amorphous PC materials, but also various other performance improvements, such as power consumption, endurance, and potential multi-bit 


\section{WILEY-VCH}

data-storage capability. As a result, an intensive effort has been devoted to understand the role of $\mathrm{N}$ dopants on the crystallization kinetics, particularly of the prototypical PC material, $\mathrm{Ge}_{2} \mathrm{Sb}_{2} \mathrm{Te}_{5}$ (GST), doped with nitrogen (NGST).

Experimental observations at a macroscopic scale for N-doped GST point to a significant alteration in crystallization kinetics for a low level of doping by nitrogen, notably resulting in a strong grain refinement, and a sizable increase in both crystallization temperature $\left(T_{c}\right)$ and the electrical resistance. In line with the increase in $T_{c}$, the incubation time for crystal nucleation, seemingly along with the growth time, increased by up to an order of magnitude with $\mathrm{N}$ doping. ${ }^{[17,19]}$ It is noted that, contrary to most cases, an increase in crystallization time was reported when a nitride layer was inserted into a GST thin film. ${ }^{[20]}$ At the same time, the $\mathrm{N}$ doping induced a substantial broadening in the distribution of nucleation times, ${ }^{[17,19]}$ indicative of the $\mathrm{N}$-induced formation of nucleation sites with very different nucleation behaviour on the microscopic scale. Computational approaches, in particular $a b$ initio molecular-dynamics (AIMD) simulations, have been recently employed to understand the effect of $\mathrm{N}$ doping at the atomic scale. Contrary to the crystallization studies of undoped GST materials, ${ }^{[21,22]}$ previous AIMD simulations for NGST failed to emulate the drastic N-induced changes in crystallization behaviour, merely revealing a structural alteration in the vicinity of $\mathrm{N}$ atoms at the molecular scale. ${ }^{[2,24]}$ Moreover, despite some promising results of mean-field theoretical approaches, ${ }^{[25,26]}$ a vivid description of the concrete microscopic processes involved in the nucleation and growth is generally very limited. Therefore, the atomistic origins responsible for the altered crystallization kinetics upon $\mathrm{N}$ doping remain unanswered, in spite of its scientific, and practical, importance. In this report, we show how a minor level of $\mathrm{N}$ doping in GST can have a decisive effect on the crystal nucleation-and-growth processes from AIMD simulations with model sizes larger than those of previous doped GST models $^{[24,27-29]}$ (see supplementary information). A microscopic mechanism governing the 


\section{WILEY-VCH}

crystallization kinetics in NGST is provided from the investigation of various aspects of doping effects on an atomic scale, from which most of the experimental observations reported so far can be comprehensively understood, presumably with implications for the physics of crystallization in other doped glasses.

In order to investigate the effect of doping on crystallization, we performed conventional annealing AIMD crystallization simulations ${ }^{[21]}$ for NGST using the Vienna ab-initio simulation package (VASP) ${ }^{[30]}$ under the generalized gradient approximation (GGA) of Perdew-Burke-Ernzerhof $(\mathrm{PBE})^{[31]}$ with a projected augmented-wave (PAW) method. ${ }^{[32]}$ Figure 1a shows the observed distribution of the onset times $\left(t_{i n c}\right)$ for the formation of stable crystalline clusters at $700 \mathrm{~K}$ (i.e. the incubation time for simulated nucleation) in undoped 180-atom GST models (whose length of one side of the cubic simulation box was $\sim 1.8 \mathrm{~nm}$ ), for which all the incubation times were within $\sim 0.3 \mathrm{~ns}$ at $700 \mathrm{~K}$. For comparison, the distribution of incubation times for the similarly sized GST models at $600 \mathrm{~K}$ is also shown in Figure 1a. Significantly, though, the inclusion of only 1.6 at.\% $\mathrm{N}$ completely prevented the formation of stable crystalline clusters throughout the whole annealing time of $\sim 3 \mathrm{~ns}$ at $700 \mathrm{~K}$ (Figure 1b). This annealing time for the NGST simulation was much longer than the observed maximum incubation time for the GST model at $700 \mathrm{~K}$, which can therefore be considered long enough for a meaningful comparison to be made between GST and NGST models; a similar behaviour was found for a model with 2.7 at. $\% \mathrm{~N}$ doping at $700 \mathrm{~K}$. Although it is still not clear whether the simulated values represent actual incubation times due to possible finite model-size effects, it becomes evident from a comparison between similarly sized GST and NGST models that, at least at $700 \mathrm{~K}$, a minor level of $\mathrm{N}$ doping can effectively disturb the occurrence of a nucleation event, resulting in a substantial increase in the incubation time for nucleation, as has been similarly observed in experiment. ${ }^{[17,19]}$ 


\section{WILEY-VCH}

The impact of $\mathrm{N}$ doping on crystal growth in GST is demonstrated in Figure 2 (see Supplementary Information for the method of crystal-growth simulations). In the model configuration shown, a single $\mathrm{N}$ atom is found to retard the propagation of a crystal plane of GST at $600 \mathrm{~K}$ by at least $\sim 0.1 \mathrm{ns,}$ which corresponds to the time scale required for about $\sim 2$ $\mathrm{nm}$ of growth from the $\mathrm{N}$-free side of the crystalline-template plane (the middle panels in Figure 2a). This result is in direct contrast to the case of undoped GST, in which no similar retardation in growth was observed (see the left panels in Figure 2a). A larger 329-atom NGST model (25\% longer along the c-axis) showed the same behaviour (see Figure S6), which further supports the above simulational results. A similar retardation of growth is observed in the NGST models annealed at $700 \mathrm{~K}$ (see the right panels in Figure 2a and Figure S6). The observed spatial extent of the (presumably transient) pinning of growth along the crystalline plane ( $\mathrm{x}-\mathrm{y}$ plane) is found to reach to a length scale (at least $>1 \mathrm{~nm}$ ) much beyond the spatial extent of the $\mathrm{N}$ complexes themselves. Although a quantitative comparison with experiments is, unfortunately, not possible due to a seeming lack of experimental crystalgrowth data in NGST, as well as simulational limitations (such as the limited model size), the retarded crystal growth inferred from experiments ${ }^{[17,19]}$ is well reproduced here.

The drastic change in the simulated nucleation times, and in growth behaviour, with less than 2 at. $\%$ of $\mathrm{N}$ doping, at least, implies that the overall structural changes (hence, the averaged structural parameters) are unable to describe the crystallization kinetics in NGST (see Table S1, Figure S1 and S2). The atomic diffusivity $(D)$, on the other hand, is one of the key parameters that control the kinetic term of the nucleation and growth probabilities in classical nucleation theory (CNT). Therefore, in the following, we first examine the Ninduced impact on atomic diffusivity in GST, as a first step in exploring the origin of the retarded nucleation-and-growth processes. 


\section{WILEY-VCH}

Figure 3a displays the calculated diffusion coefficients of elements in GST and NGST at 700K, exhibiting a seemingly universal, element-dependent, slowdown with increased levels of doping in NGST. On the other hand, an analysis of the probability distribution (Figure 3b), and spatial dependence (Figure 3c), of atomic displacements reveals that this conventional interpretation is not at all sufficient, but one needs additionally to consider the emergence of collective atomic movements, which is manifested in the formation of mobile $\mathrm{N}$-free, and immobile, N-centred clusters (Figure 3c). These data indicate that the averaged value of $D$ for each atomic species underestimates the actual slowdown of atoms adjacent to $\mathrm{N}$ atoms (at most by $\sim 50 \%$ ), and a spatial consideration (i.e. the localized nature of dynamic slowdown) is required for a precise description of atomic dynamics in NGST. It is noted that an $\mathrm{N}_{2}$ molecule (observed in the 10 at. \% N-doped GST model) showed the highest diffusivity due to its negligible chemical interactions with the host GST elements (see Figure S5), whose role in crystallization, in particular, for heavily doped cases may be of interest for a further study. ${ }^{[33]}$ In any case, within the framework of CNT, the N-induced slowdown in dynamics should lead to a corresponding decrease in nucleation and growth probabilities. Therefore, the retarded crystallization can be partially attributed to the decreased atomic mobility caused by $\mathrm{N}$ dopants. In a quantitative sense, though, the large delay in the simulated nucleation time of NGST, at least an order of magnitude larger than that of GST, may require further explanation, presumably beyond the dynamical slowdown. As a passing remark, the decrease in atomic mobility may be associated with the improved endurance of PC memory (PCM) devices with $\mathrm{N}$ doping, ${ }^{[20]}$ as this could suppress the elemental segregation, ${ }^{[34]}$ one of the known failure mechanisms of PCM cells.

The analysis of atomic trajectories, and of the local medium-range structural order, during annealing at $700 \mathrm{~K}$ reveals that, indeed, there exists another important crystallization parameter of the local-structural heterogeneity that significantly influences both the 


\section{WILEY-VCH}

nucleation (Figure 1c) and growth processes (Figure 2b). More specifically, due to a topological mismatch (i.e. different bond angles and bond lengths) in the local coordination between $\mathrm{N}$ dopants and GST host elements, the growth of a (crystalline-like) planar structure, which has been identified as an intermediate step for the fast 'normal' growth of cubic clusters in GST, ${ }^{[2]}$ was frustrated by severely distorted 4-fold (or higher-order) rings around $\mathrm{N}$-complexes (Figure 1c) that prevent a crystalline-like cluster (represented as spikes in Figure 1b) from growing beyond the critical-nucleus size (5-10 cubes for this model size ${ }^{[22]}$ ). In fact, this observation conforms to the current consensus that the ultrafast crystallization of GST could be facilitated by the cooperative atomic movements, driven by near-identical local structures (i.e. a defective octahedral coordination) of GST elements. ${ }^{[21,22,35]}$; $\mathrm{N}$ dopants with a tetrahedral, or trigonal, local coordination frustrate the cooperativeness in atomic motions, thereby interrupting the nucleation process. Such kinetic constraints similarly affected the crystal-growth process at the amorphous-crystalline interfaces (Figure 2b). The significant influence of topological incompatibility on the static structure of crystallized GST is also evident from Figure 2c, where the (distorted) rock-salt structure of metastable crystalline GST suffers from a severe lattice distortion near the $\mathrm{N}$ atom. Such structural imperfections due to $\mathrm{N}$ dopants should cause an increase of system energy. ${ }^{[23,24]}$ According to a theoretical consideration based on CNT (see supplementary information for details), the nucleation probability is likely to diminish, due to an increase in the energy barrier for nucleation, in such a case, i.e. when a dopant (or more generally a cluster of dopant complexes) forms heterogeneous interfaces with a host matrix, which is the case expected for $\mathrm{N}$ dopants in GST. It is also suggested that the more (or larger) $\mathrm{N}$ clusters that a subcritical nucleus contains, the less probable becomes the occurrence of the nucleation event. Putting these simulational and theoretical observations together, we can conclude that one of the central consequences of $\mathrm{N}$ doping would be the formation of constrained nucleation, and growth, sites at the expense of normal sites, the former being more sluggish in speed, yet demanding a higher activation 


\section{WILEY-VCH}

energy, for transformation than the latter. The formation (or coexistence) of such sites is supported by various experimental observations, including the elongated (average) nucleation times, accompanying the broadening in their distributions, ${ }^{[17,19]}$ and the change in the lattice constant. $^{[13]}$

To support further the findings so far, as well as to get more general ideas on factors affecting crystallization in doped GST, we studied more dopant species as diverse as elements over Groups III-VI of the Periodic Table (Figure 4). The simulated diffusivities of dopants showed a broad distribution in their values with up to an order of magnitude difference at $600 \mathrm{~K}$, and the topology of the local structure in GST was either (defective) octahedral, tetrahedral, trigonal geometry, or their mixtures. Significantly, we could immediately notice discernible trends that: i) all of the experimentally-identified "constraining" dopants (indicated as larger circles in Figure 4) have structural topologies different from a defective octahedral configuration; and ii) the dopants in the least mobile group (viz. N, Si, C, and B) all belong to the class of constraining dopants. These data, thus, not only support the proposed mechanisms governing the slow crystallization kinetics in NGST, i.e. the dissimilarity in topology and slowdown in diffusivity, but also imply that the retardation mechanism of $\mathrm{N}$ doping may be similarly applicable to those for the other constraining dopants, in particular $\mathrm{Si}$, $\mathrm{C}$, and $\mathrm{B}$. On the other hand, considering the cases of $\mathrm{O}, \mathrm{Al}$, and $\mathrm{Ga}$, where the constraining effect is poorly describable in terms of the low $D$, a non-octahedral topology is the only universal character of constraining dopants, perhaps indicative of the central importance of the topological aspect over the other factors. Another noteworthy observation from Figure 4 is that there exist apparent correlations between the studied parameters. For instance, the impact of dopants on crystallization speed in GST may be guessed from the strength of covalent bonds formed between dopants and host elements, since the bond strength seems to be an approximate measure of diffusivity according to the $\tau-D$ correlation in Figure 4. A reasonable 


\section{WILEY-VCH}

explanation for the $\tau-D$ correlation (seemingly with an inverse power-law dependence) emerges from the observed formation of tightly-bound 'molecular' cages around dopants that eventually disturbed hopping-induced diffusion. The previous argument of doping-induced modification in crystallization in terms of bond strength ${ }^{[36]}$ (or analogously bond enthalphy ${ }^{[26]}$ ) may be, therefore, more-or-less rationalized by the correlated change in diffusivity. The other signature of constraining dopants of a small atomic size (the inset of Figure 4) can be similarly understood by their non-octahedral coordination, often together with their low diffusivities. The existence of such correlations thus allows many dopant characteristics, including those proposed to be relevant by others, ${ }^{[26,36,37]}$ to be explainable within our retardation-mechanism scheme.

So far, we have focused on the microscopic parameters associated with the 'kineticallyconstrained' transformation in NGST. From now on, we discuss its implications on a larger scale, possibly insightful for understanding the macroscopic crystallization behaviour. First of all, a gradual switching from normal to constrained sites with increasing $\mathrm{N}$ concentration can generally explain the slowdown in crystallization speed ${ }^{[17]}$ and the increase in $T_{c},{ }^{[15]}$ which is a known reason for the improved data-retention property of NGST-based PCM devices. As more severely constrained sites are being generated with increasing $\mathrm{N}$ concentrations, $\mathrm{a}$ gradual decrease in the effective crystallization volume must be expected. Accordingly, the residual non-crystallized volume fractions, ${ }^{[18]}$ together with a possible disorder-induced electronic localization in crystalline grains as a result of constrained nucleation and growth events, could lead to an increased electrical resistance of crystallized GST upon N doping, and a consequent reduction in power consumption in PCM cells. ${ }^{[38]}$

Another interesting experimental observation with $\mathrm{N}$ doping is the grain-refinement phenomenon. ${ }^{[13,14,39]}$ The segregation of nitrogen atoms at grain boundaries ${ }^{[15]}$ has often been assumed to be responsible, yet with little evidence; therefore, the question of what is the 


\section{WILEY-VCH}

microscopic origin of grain refinement is still not answered. Thus, we present here some implications from our simulational results in favour of this hypothesis, at least partially. Although a lack of information on $\mathrm{N}$ distributions available from experiments precludes any detailed discussion, we can schematically describe the grain-refinement phenomenon from a clustered distribution of $\mathrm{N}$ dopants, originating from an exceptionally long lifetime $\left(\tau_{\mathrm{Ge}-\mathrm{N}}\right)$ of Ge-N bonds (Figure 5a). The observed process of cluster formation is clearly illustrated in Figure 5b; once $\mathrm{N}$ complexes form a cluster after random diffusion, they tend to spend more time in the cluster due to the high value of $\tau$ (Figure $5 \mathrm{c}$ ), which then substantially increases the probability of forming a larger cluster by absorbing other $\mathrm{N}$ atoms. Among possible $\mathrm{N}$ distributions (Figure 5d), therefore, we believe that the intermediate case between the random and completely clustered distributions may be most likely, although the degree of clustering in question should vary, depending on the thermal history that determines the evolution of $\tau$ and $D$. In the case of undoped GST, the growth of a nucleus is restricted only by the nearby growing nuclei, i.e., the relative ratio of nucleation to growth rates is the only important parameter. ${ }^{[40]}$ On the other hand, in the case of NGST, the N-induced pinning in growth (Figure 2), together with the help of the schematic description of dopant distributions in Figure 5d, suggests that the presence of large clusters of $\mathrm{N}$ complexes imposes additional restrictions on the growth of individual supercritical nuclei (or grains), which should result in a smaller average grain size than otherwise would be the case. An empirical, yet indirect, piece of evidence supporting such a clustering-driven, grain-refinement mechanism is that grain refinement was commonly observed for most of the constraining dopants ${ }^{[37,41-44]}$ which showed, at the same time, a tendency of clustering, either observed from simulations (Figure S4) or predicted from their large values of $\tau$.

In summary, the topological, and dynamic, aspects of $\mathrm{N}$ doping, together with the spatial dopant distribution, were found to critically influence the crystallization kinetics of NGST. 


\section{WILEY-VCH}

The obtained knowledge, as to the relationships between crystallization kinetics and dopant characteristics, may be useful, not only for selecting dopants (possibly in silico) for optimizing the performance of PCM devices, but also for understanding the effects of doping on crystallization in other glasses. For instance, many aspects of the empirical criteria found in the crystallization of metallic glasses ${ }^{[45]}$ are analogous to those found in this study. The idea of a local kinetic constraint with doping, therefore, may be applicable to, and hence can be tested in, other glass systems, with much broader implications.

\section{Supporting Information}

Supporting Information is available from the Wiley Online Library or from the authors.

\section{Acknowledgements}

We acknowledge financial support from the Engineering and Physical Sciences Research Council (UK). The AIMD simulations were performed using the Cambridge High-Performance Computing Facility (Darwin). We thank the Data Storage Institute, Agency for Science, Technology and Research (Singapore) for the use of their facilities to fabricate and characterize the test samples. Discussions with Prof. A.L. Greer are gratefully acknowledged.

Received: ((will be filled in by the editorial staff))

Revised: ((will be filled in by the editorial staff)) Published online: ((will be filled in by the editorial staff))

[1] X. F. Duan, Y. Huang, Y. Cui, J. F. Wang, C. M. Lieber, Nature 2001, 409, 66.

[2] J. M. D. Coey, M. Venkatesan, C. B. Fitzgerald, Nat. Mater. 2005, 4, 173.

[3] F. Wang, Y. Han, C. S. Lim, Y. Lu, J. Wang, J. Xu, H. Chen, C. Zhang, M. Hong, X. Liu, Nature 2010, 463, 1061.

[4] K. H. Behrndt, J. Vac. Sci. Technol. 1970, 7, 385.

[5] W. Felsch, Z. Physik 1966, 195, 201.

[6] A. L. Greer, Science 1995, 267, 1947.

[7] M. Wuttig, N. Yamada, Nat. Mater. 2007, 6, 824.

[8] D. Loke, T. H. Lee, W. J. Wang, L. P. Shi, R. Zhao, Y. C. Yeo, T. C. Chong, S. R. Elliott, Science 2012, 336, 1566. 


\section{WILEY-VCH}

[9] M. Cassinerio, N. Ciocchini, D. Ielmini, Adv. Mater. 2013, 25, 5975.

[10] D. Loke, J. M. Skelton, W. J. Wang, T. H. Lee, R. Zhao, T. C. Chong, S. R. Elliott, Proc. Natl. Acad. Sci. U.S.A. 2014, 111, 13272.

[11] D. Kuzum, R. G. D. Jeyasingh, B. Lee, H.-S. P. Wong, Nano Lett. 2012, 12, 2179.

[12] T. H. Lee, D. Loke, W. J. Wang, K. J. Huang, S. R. Elliott, Adv. Mater. 2014, 26, 7493.

[13] T. H. Jeong, M. R. Kim, H. Seo, J. W. Park, C. Yeon, Jpn. J. Appl. Phys. 2000, 39, 2775.

[14] H. Kolpin, D. Music, G. Laptyeva, R. Ghadimi, F. Merget, S. Richter, R. Mykhaylonka, J. Mayer, J. M. Schneider, J. Phys.: Condens. Matter 2009, 21, 435501.

[15] R. Kojima, S. Okabayashi, T. Kashihara, K. Horai, T. Matsunaga, E. Ohno, N. Yamada, T. Ohta, Jpn. J. Appl. Phys. 1998, 37, 2098.

[16] B. Liu, Z. Song, T. Zhang, J. Xia, S. Feng, B. Chen, Thin Solid Films 2005, 478, 49.

[17] R. M. Shelby, S. Raoux, J. Appl. Phys. 2009, 105, 104902.

[18] I. Yang, K. Do, H.-J. Chang, D.-H Ko, H. Sohn, J. Electrochem. Soc. 2010, 157, H483.

[19] K. Darmawikarta, S. Raoux, P. Tchoulfian, T. Li, J. R. Abelson, S. G. Bishop, J. Appl. Phys. 2012, 112, 124907.

[20] C. Trappe, B. Bechevet, B. Hyot, O. Winkler, S. Facsko, H. Kurz, Jpn. J. Appl. Phys. 2000, 39, 766.

[21] J. Hegedus, S. R. Elliott, Nat. Mater. 2008, 7, 399.

[22] T. H. Lee, S. R. Elliott, Phys. Rev. Lett. 2011, 107, 145702.

[23] S. Caravati, D. Colleoni, R. Mazzarello, T. D. Kuhne, M. Krack, M. Bernasconi, M. Parrinello, J. Phys.: Condens. Matter 2011, 23, 265801.

[24] E. Cho, S. Han, D. Kim, H. Horii, H. S. Nam, J. Appl. Phys. 2011, 109, 043705.

[25] M. H. R. Lankhorst, J. Non-Cryst. Solids 2002, 297, 210.

[26] S. Raoux, M. Salinga, J. L. Jordan-Sweet, A. Kellock, J. Appl. Phys. 2007, 101, 044909.

[27] Z. M. Sun, J. Zhou, H. J. Shin, A. Blomqvist, R. Ahuja, Appl. Phys. Lett. 2008, 93, 241908. 


\section{WILEY-VCH}

[28] K. B. Borisenko, Y. X. Chen, S. A. Song, D. J. H. Cockayne, Chem. Matter. 2009, 21, 5244.

[29] J. M. Skelton, S. R. Elliott, J. Phys.: Condens. Matter 2013, 25, 205801.

[30] G. Kresse, J. Hafner, Phys. Rev. B 1993, 47, 558.

[31] J. P. Perdew, K. Burke, M. Ernzerhof, Phys. Rev. Lett. 1996, 77, 3865.

[32] P. E. Blöchl, Phys. Rev. B 1994, 50, 17953.

[33] A. V. Kolobov, P. Fons, B. Hyot, B. Andre, J. Tominaga, Y. Tamenori, H. Yoshikawa, K. Kobayashi, Appl. Phys. Lett. 2012, 100, 061910.

[34] T.-Y. Yang, J.-Y. Cho, Y.-J. Park, Y.-C. Joo, Acta Mater. 2012, 60, 2021.

[35] T. Matsunaga, N. Yamada, Jpn. J. Appl. Phys. Part 1 2002, 41, 1674.

[36] S. Wei, J. Li, X. Wu, P. Zhou, S. Wang, Y. Zheng, L. Chen, F. Gan, X. Zhang, G. Li, Opt. Express 2007, 15, 10584.

[37] X. Zhou, L. Wu, Z. Song, F. Rao, M. Zhu, C. Peng, D. Yao, S. Song, B. Liu, S. Feng, Appl. Phys. Lett. 2012, 101, 142104.

[38] Y. N. Hwang, S. H. Lee, S. J. Ahn, S. Y. Lee, K. C. Ryoo, H. S. Hong, H. C. Koo, F. Yeung, J. H. Oh, H. J. Kim, W. C. Jeong, J. H. Park, H. Horii, Y. H. Ha, J. H. Yi, G. H. Koh, G. T. Jeong, H. S. Jeong, K. Kim, IEEE International Electron Devices Meeting (IEDM), 2003, 893.

[39] K. Do, H. Sohn, D. H. Ko, J. Electrochem. Soc. 2007, 154, H867.

[40] A. L. Greer, Mater. Sci. Eng. A 1991, A133, 16.

[41] B. Qiao, J. Feng, Y. Lai, Y. Ling, Y. Lin, T. Tang, B. Cai, B. Chen, Appl. Surf. Sci. 2006, $252,8404$.

[42] N. Matsuzaki, et al. IEDM Tech. Dig. 2005, 757.

[43] C. Sandhya, A. Bastard, L. Perniola, J. C. Bastien, A. Toffoli, E. Henaff, A. Roule, A. Persico, B. Hyot, V. Sousa, B. De Salvo, G. Reimbold, In IEEE International Reliability Physics Symposium (IRPS), 2012, 6C.3.1. 


\section{WILEY-VCH}

[44] Y. Lu, Z. Zhang, S. Song, X. Shen, G. Wang, L. Cheng, S. Dai, Z. Song, Appl. Phys. Lett. 2013, 102, 241907.

[45] W. H. Wang, Prog. Mater. Sci. 2007, 52, 540.

[46] T. H. Jeong, H. Seo, K. L. Lee, S. M. Choi, S. J. Kim, S. Y. Kim, Jpn. J. Appl. Phys. 2001, 40, 1609. 


\section{WILEY-VCH}

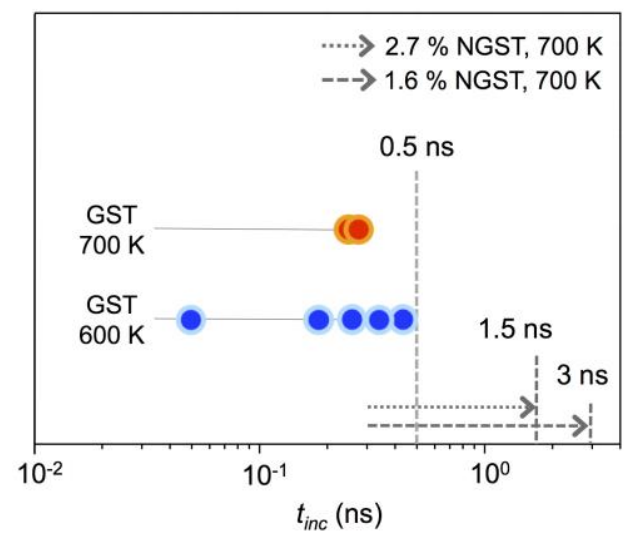

b

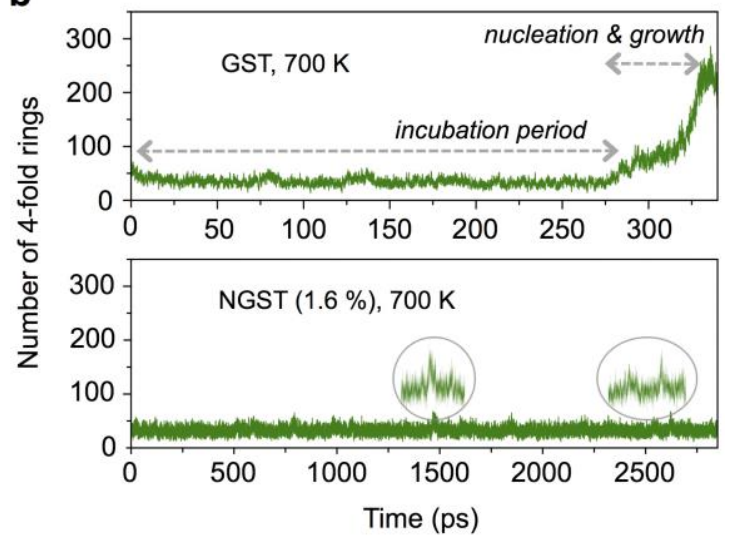

C

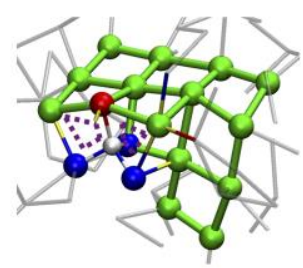

$(\mathrm{Ge} / \mathrm{Sb})_{4} \mathrm{~N}$

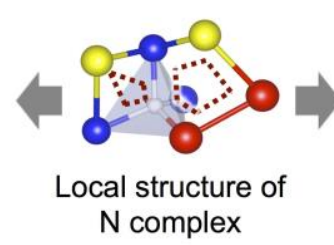

$\mathrm{N}$ complex

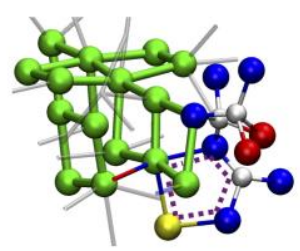

$\left[(\mathrm{Ge} / \mathrm{Sb})_{4} \mathrm{~N}_{2}-\mathrm{Ge}_{3} \mathrm{~N}\right.$

Figure 1. Effects of $\mathrm{N}$ atoms on simulated nucleation. a) Distribution of incubation times $\left(t_{\text {inc }}\right)$ for nucleation generated from GST models at $700 \mathrm{~K}$. For comparison, the distribution of incubation times at $600 \mathrm{~K}$, some of which are taken from the simulations in [22], is also shown. b) Evolution of the number of 4-fold rings during annealing at $700 \mathrm{~K}$ for models of GST and NGST (1.6 at.\% N). The circled, magnified spikes in the lower panel indicate the formation of transient crystalline clusters in the NGST model. c) Snapshots illustrating a retardation mechanism due to a topological mismatch. Atoms in the crystalline-like cluster are coloured as green, otherwise coloured as Ge (blue), Sb (red), Te (yellow) and N (silver). 


\section{WILEY-VCH}

a
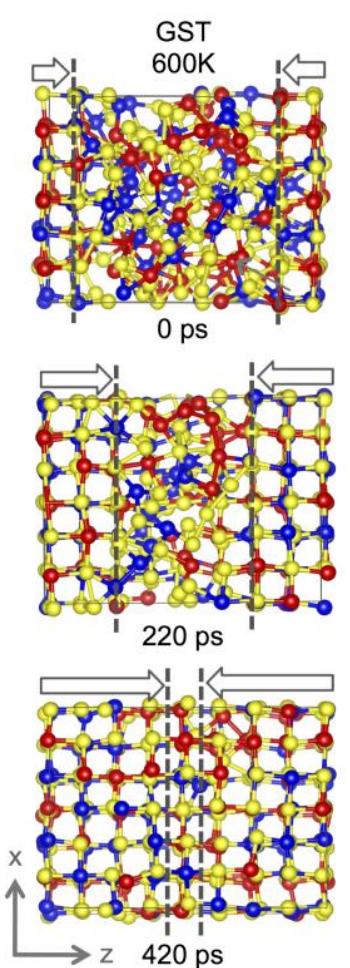
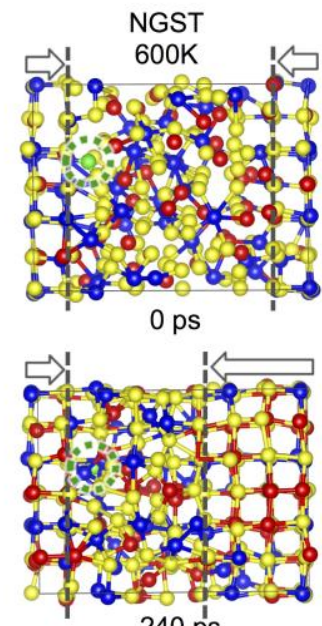

240 ps

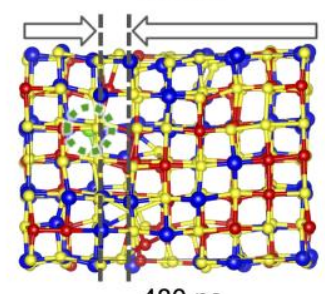

$480 \mathrm{ps}$
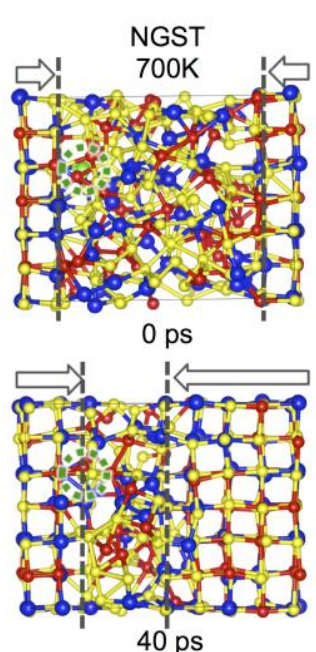
$40 \mathrm{ps}$

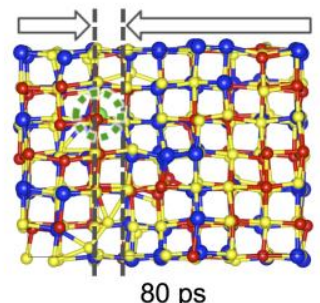

b

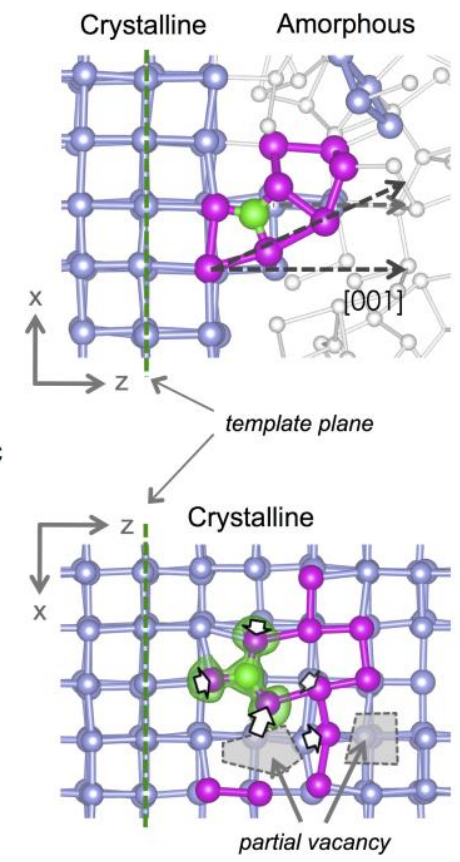

Figure 2. Impact of an $\mathrm{N}$ dopant atom (shown as green) on simulations of templated crystalline growth in GST. a) Comparison of the relative growth of crystalline regions from the template planes in GST $(600 \mathrm{~K})$ and NGST $(600 \mathrm{~K}$ and $700 \mathrm{~K})$ models, represented by the arrows. Atoms are coloured as Ge (blue), $\mathrm{Sb}$ (red), Te (yellow) and N (green). b) Atomic configuration near the $\mathrm{N}$ atom (green) during crystallization at $600 \mathrm{~K}$, the dashed arrow showing the direction of the $\mathrm{N}$-induced crystallization direction, relative to the [001] direction of crystallization for the rest of the model. c) Fully crystallized NGST model at $600 \mathrm{~K}$, the arrows showing the local atomic distortions around the N dopant atom. Significantly distorted atoms are coloured as purple, and partial vacancies are also indicated. 

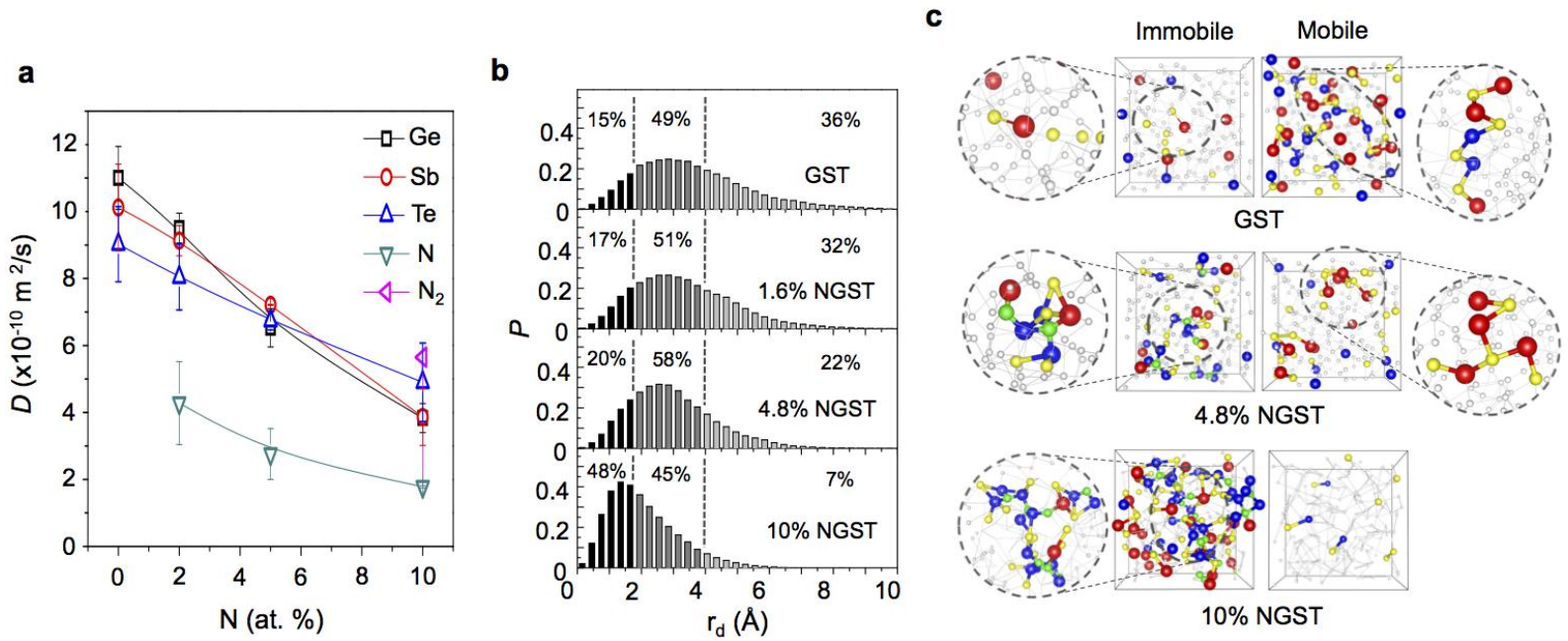

Figure 3. Local slowdown in dynamics induced by $\mathrm{N}$ doping. a) Diffusion coefficients at 700 $\mathrm{K}$, averaged over each type of atom, as a function of $\mathrm{N}$ concentration. Due to the unknown density of NGST, the diffusion coefficients were obtained from simulations with two different (low and high) model densities of $5.84 \mathrm{~g} / \mathrm{cm}^{3}$ and $6.11 \mathrm{~g} / \mathrm{cm}^{3}$, respectively. b) Probability distribution of atomic displacements, $P$, within 25 ps of annealing at $700 \mathrm{~K}$. About 3200 origin configurations for each model were sampled to improve the statistics. c) Snapshots highlighting mobile and immobile atoms and their clusters. Mobile atoms are defined to travel a certain distance beyond their next nearest neighbours $(\sim 4 \AA)$ in 25 ps of annealing, while immobile atoms are those whose maximum displacement, $r_{\mathrm{d}}$, is less than $1.8 \AA$, as indicated in b). Atoms are coloured as Ge (blue), $\mathrm{Sb}$ (red), Te (yellow) and N (green). 


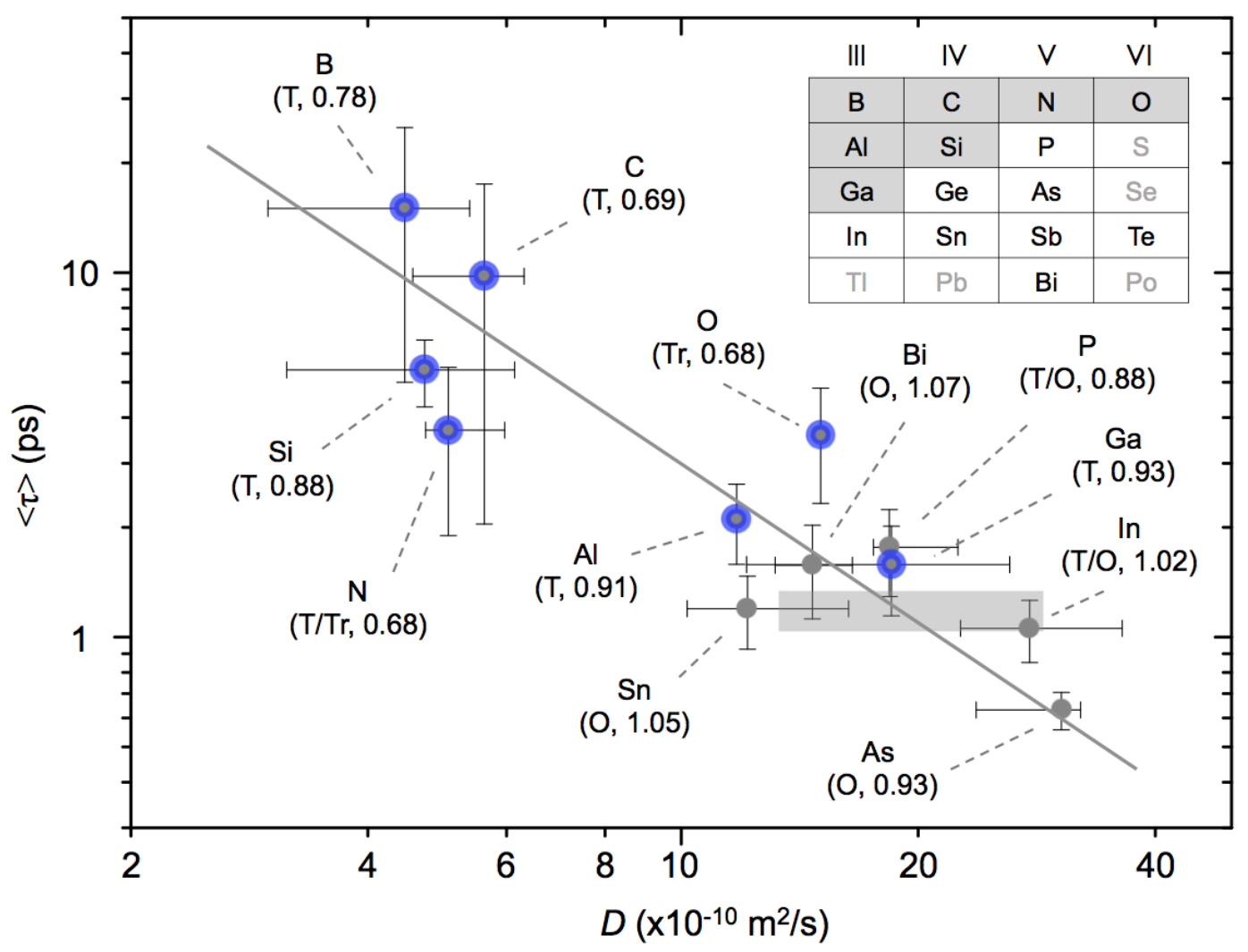

Figure 4. Dynamical and structural characteristics of dopants (Groups III-VI) in GST at 600 K. Constraining dopants that tend to increase $T_{c}$ with doping (i.e. $\mathrm{N}, \mathrm{Si}^{[41]}, \mathrm{O}^{[46]}, \mathrm{C}^{[37]}, \mathrm{B}^{[43]}$, $\mathrm{Ga}^{[44]}$, and $\mathrm{Al}^{[36]}$ ) are highlighted by large blue circles. The topology of the local structure and the mean bond length for each dopant are also indicated in the parentheses: $\mathrm{T}$, Tr, or O denote tetrahedral, trigonal or (defective) octahedral geometries of dopant complexes, respectively; the number is the value of the ratio $R_{\text {dopant }} / R_{G e / S b}$. We note that the experimental results for the B dopant refer to a GeTe host and for Ga to $\mathrm{a} \mathrm{Ge}_{3} \mathrm{Sb}_{2} \mathrm{Te}_{5}$ host, while the host composition for all the other dopants was GST. No report has been published for P and As doping, as far as we are aware. The gray square area denotes the range of values for $\tau$ and $D$ for $\mathrm{Ge}, \mathrm{Sb}$ or $\mathrm{Te}$ atoms. The least-squares fitted power-law exponent for the $\tau_{\text {mean }}$ vs $D$ curve is $-1.4 \pm 0.6$. The inset displays the investigated dopants, with constraining elements in darker backgrounds. 
a

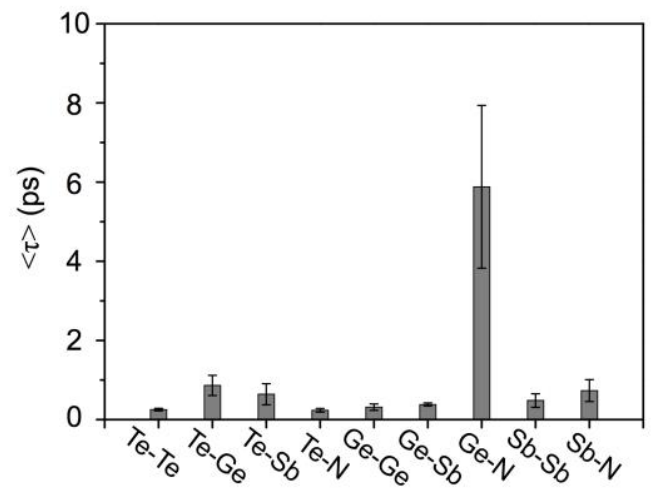

C

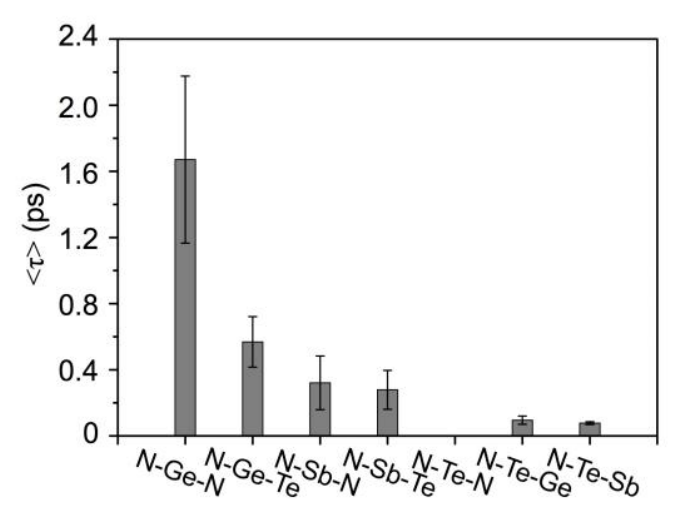

b

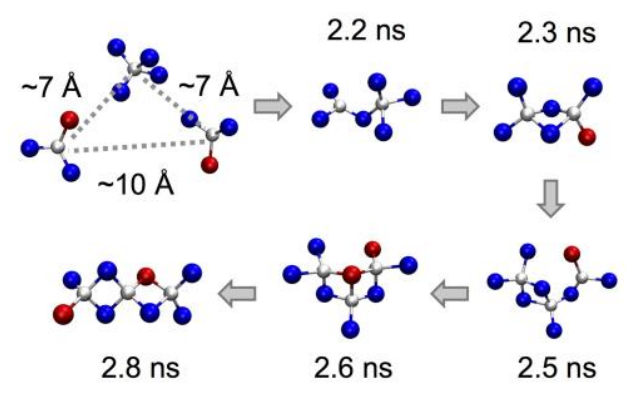

d

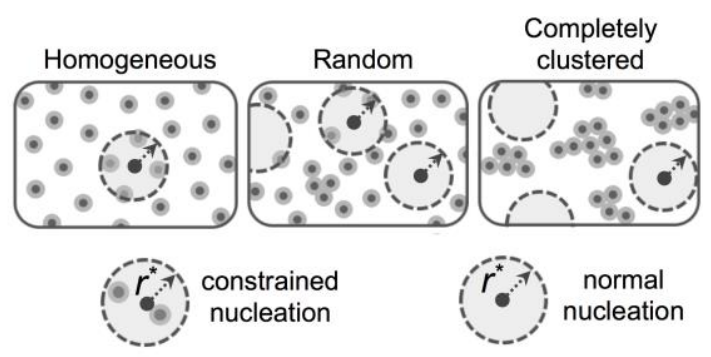

Figure 5. Clustering tendency of $\mathrm{N}$ atoms and its impact on crystallization kinetics. a) Mean bond lifetimes for different types of atom pairs, calculated during annealing at $700 \mathrm{~K}$ (1.6 at.\% $\mathrm{N}$ doped model). b) Formation of a cluster of $\mathrm{N}$ complexes during annealing simulations. Atoms in the $\mathrm{N}$ complexes are coloured as Ge (blue), Sb (red), and N (silver). c) Mean bond lifetimes for different triplet N-bonding configurations. d) A schematic description of representative dopant distributions. A random distribution may be an intermediate, yet presumably the most likely, distribution in the absence of notable inter-dopant interactions or clustering tendency. $r^{*}$ denotes the critical size of a nucleus. 